\title{
Stents coronarios y cirugía no cardiaca: estrategias de manejo actual
}

\author{
PAULO CARRASCO G., RODRIGO LÓPEZ B., \\ GUILLERMO LEMA F.
}

\section{Coronary stents and non-cardiac surgery: current management strategies}

The care of patients with coronary stents in the perioperative period of non-cardiac surgery requires anesthesiologists, surgeons and cardiologists. The management of dual antiplatelet therapy should be handled depending on type and urgency of the surgery, the risk of bleeding, type of coronary stent implanted, time from placement and the risk of stent thrombosis. For patients identified as high risk of stent thrombosis, surgery should be planned at hospitals with cardiac catheterization facilities. These patients require postoperative monitoring and antiplatelet therapy should be restarted immediately. Stent thrombosis should be recognized early and treated aggressively with percutaneous coronary intervention.

(Rev Med Chile 2011; 139: 787-793).

Key words: Angioplasty, balloon, coronary; Coronary artery disease; Stents; Thoracic surgery.

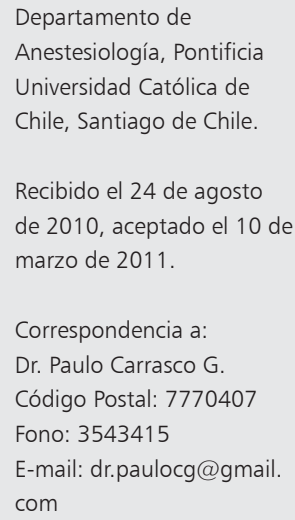

E $\mathrm{n}$ los últimos años ha existido un aumento importante en el número de pacientes que reciben stents coronarios (SC), realizándose anualmente en Estados Unidos de Norteamérica (USA) más de 1,5 millones de intervenciones coronarias percutáneas (ICP), 90\% de ellas con implantación de al menos un SC, trasformándose en la intervención de elección para el tratamiento de la enfermedad coronaria por sobre la cirugía de revascularización miocárdica ${ }^{1}$.

Es esperable que un grupo de estos pacientes se presenten para cirugía no cardiaca, alcanzando hasta $5 \%$ dentro del primer año post $\mathrm{SC}^{2}$. El equipo médico involucrado en el perioperatorio de estos pacientes (cardiólogo, anestesiólogo y cirujano) deben conocer el tratamiento con antiagregante plaquetarios, los riesgos de trombosis del SC y los riesgos de hemorragia como consecuencia de la cirugía. El propósito de esta revisión es repasar algunos aspectos de esta situación clínica y entregar algunas posibles estrategias de manejo.

\section{Stents coronarios, evolución y antiagregación plaquetaria}

En respuesta a la alta incidencia de re-estenosis con las angioplastías coronarias con balón, se introdujo la técnica de SC. Los primeros en usarse fueron los stents metálicos o stents mo medicados (SNM), con la finalidad de evitar la retracción elástica del lumen y la oclusión del vaso, disminuyéndola en 20-30\% en relación a la plastía con balón ${ }^{3}$. Antes de la implementación de la antiagregación plaquetaria dual, los SNM presentaban una elevada tasa de trombosis aguda, situación que prácticamente desapareció con la recomendación de usar 4 a 6 semanas aspirina y clopidogrel, permitiendo la total re-endotelización y evitando la eventual trombosis del SC $\mathrm{S}^{5}$. Sin embargo, este dispositivo causa hiperplasia de la neo íntima y re-estenosis en $12-20 \%$ de los pacientes a los 6 meses, llegando hasta $80 \%$ en subgrupos específicos (diabéticos o insuficientes renales), haciendo frecuente la necesidad de reintervenir ${ }^{4}$. 
Los stents medicados (SM) se desarrollaron para enfrentar el problema de la re-estenosis, a través de la liberación de fármacos antineoplásicos o inmunosupresores desde su superficie. Estos fármacos inhiben la proliferación de músculo liso y la hiperplasia endotelial, disminuyendo el riesgo de re-estenosis en $74 \%{ }^{6}$. Sin embargo, al inhibir la endotelización la superficie metálica del SC queda sin protección, con potencial trombogénico por un período de tiempo hasta ahora desconocido, existiendo reportes post-mortem de pacientes sin reendotelización hasta 4 años de instalado el SC ${ }^{7}$.

La trombosis precoz del SC ( $<30$ días) habitualmente está relacionada con problemas técnicos de la instalación (expansión insuficiente o tamaño inadecuado) y corresponde a $1 \%$ de todos los $\mathrm{SC}^{8}$. La trombosis tardía es de baja incidencia en stents no medicados y en SM es entre 0,5 y $3,1 \%{ }^{9}$, ocurriendo habitualmente en relación a la terapia antiplaquetaria suspendida o incompleta; así en pacientes que suspenden el clopidogrel ocurre en promedio antes de 30 días y si la suspensión es de clopidogrel y aspirina el promedio es de sólo 7 días ${ }^{10}$.

La trombosis tardía del SC es un proceso agudo y potencialmente catastrófico, que no da tiempo para compensar el flujo coronaria por medio de circulación colateral, causando infarto en 25 a $65 \%$ de los pacientes, arritmias y una mortalidad entre 15 y $45 \%$ \% $910,34,35,36$.

Desafortunadamente no disponemos de exámenes que determinen si los SC se han reendotelizado y por lo tanto permanecen en riesgo de trombosis. Existen múltiples factores de riesgo para que se produzca trombosis del SC, tanto dependientes del paciente, del procedimiento o del stents $^{12,32,34,37,38}$ (Tabla 1). La discusión se concentra principalmente en la duración adecuada del tratamiento antiagregante plaquetario dual, puesto que este es el factor predictor más importante de trombosis del $\mathrm{SC}^{10,34,39}$.

\section{Stents y cirugía no cardiaca}

El proceso de trombosis del SC es un fenómeno multifactorial, requiriéndose la combinación de drogas que actúen a distintos niveles para prevenir la activación plaquetaria. La combinación más común es aspirina (inhibidor TxA2) y clopidogrel (inhibidor receptor ADP).
En un extenso estudio prospectivo multicéntrico, Iakovou y cols mostraron que el riesgo de trombosis tardía es 90 veces mayor en pacientes con SM que discontinuaron prematuramente la antiagregación en relación a los que no lo hicieron ${ }^{8}$. Un estudio retrospectivo realizado por Banerjee y cols concluyó que el uso de clopidogrel por más de 12 meses después de ICP se asoció a menor mortalidad independiente de los otros factores de riesgo ${ }^{16}$.

La suspensión de la terapia antiagregante puede causar "efecto de rebote" e hipercoagulabilidad. La suspensión de aspirina aumenta los niveles de ciclooxigenasa- 1 y tromboxano $\mathrm{A} 2$, retornando la función plaquetaria a la normalidad a los 3 días en $50 \%$ y a los 4 días en $80 \%{ }^{11}$. La suspensión brusca de clopidogrel causa un estado proinflamatorio y protrombótico, así Ho y cols reportan un aumento en los eventos cardiovasculares en pacientes con menos de 90 días de suspensión de clopidogrel ${ }^{12}$. La cirugía por sí sola también causa un estado de hipercoagulabilidad por varias semanas, en parte por aumento de la actividad plaquetaria, vasoespasmo y aumento de mediadores inflamatorios ${ }^{13}$.

Tabla 1. Factores de riesgo de trombosis de stent coronarios

Factores del paciente:
- Síndrome coronario agudo
- Mala función ventricular
- Diabetes Mellitus
- Insuficiencia renal
- Hipercoagulabilidad (cáncer, cirugía, etc.)
Factores del procedimiento:
- Stent traslapados
- Disección residual
- Expansión incompleta stent
- Técnica "crush"
- Oclusión rama lateral
Anatomía coronaria:
- Tamaño del vaso
- Stent en tronco coronaria izquierda
- Lesión larga
- Enfermedad multivaso
- Oclusión total vaso
- Bifurcación
- Características de placas
Factores del stent:
Factores antitrombóticos o anticoagulantes:
- Suspensión antiagregantes
- Resistencia antiplaquetarios
- Inhibición de la agregación


En pacientes con SNM sometidos a cirugía no cardiaca tres semanas después de la instalación de los SC, Sharma y cols comunicaron una mortalidad de $85 \%{ }^{14}$; sin embargo, Wilson y cols reportaron sólo 3\% cuando el tiempo de inserción del SNM fue mayor a 6 semanas ${ }^{15}$.

Un estudio retrospectivo reciente con 900 pacientes seguidos por 15 años, Nuttall y cols encontraron $10,5 \%$ de eventos cardiacos mayores si la cirugía no cardiaca se realizaba antes de 30 días de puesto el SNM, 3,8\% entre 31-90 días y 2,8\% si era $>90$ días. La cirugía de emergencia tuvo mayores tasas, con $11,7 \%$ versus $4,4 \%$. Concluyeron, que el riesgo de eventos cardiovasculares mayores disminuye en forma importante después de 90 días de implantado un $\mathrm{SNM}^{18}$.

Rabbits y cols revisaron retrospectivamente a 520 pacientes con SM sometidos a cirugía no cardiaca, encontrando que la tasa de eventos cardiacos mayores fue de $6,4 \%$ si la ICP fue antes de 90 días, 5,7\% entre $91-180$ días y $3,3 \%$ si fue más de 1 año. No se observó sangrado significativo mayor asociado a tratamiento antiagregante dual, concluyéndose que el principal factor de riesgo en pacientes con SM no corresponde al tiempo transcurrido entre la instalación del SC y la cirugía, sino que más bien a la antiagregación dual ${ }^{19}$.

En relación a esta evidencia, la American Heart Association (AHA) sugiere mantener la terapia dual al menos por 4-6 semanas en caso de SNM y por al menos 12 meses en caso de SM. Agrega además, que se debe mantener la terapia dual perioperatoria más allá del año en los pacientes donde las consecuencias de una trombosis tardía pueden ser fatales ${ }^{17}$ o que tienen factores de riesgo para trombosis tardía, como mala función ventricular, stents múltiples, SC traslapados o en bifurcaciones, diabetes e insuficiencia renal, entre otros $^{10-12}$.

Es importante que los pacientes y los médicos reconozcan que el plazo de 1 año, sugerido por la AHA en cuanto a postergar una cirugía electiva post SM no garantiza ausencia de eventos en el perioperatorio.

\section{Riesgo de hemorragia en tratamiento antiagregante dual}

La terapia antiagregante plaquetaria dual no está exenta de riesgos. Múltiples estudios evalúan el riesgo de sangrado quirúrgico en pacientes con antiagregación plaquetaria, la mayoría de ellos en cirugía cardiaca, asociándose el uso aspirina a un bajo riesgo de sangrado ${ }^{41}$, pero el uso combinado con tienopiridinas resulta en mayores sangrados, transfusiones, más días de ventilación mecánica y de estadía en UCI ${ }^{32,42}$.

En cirugía no cardiaca, el riesgo de sangrado quirúrgico asociado a tratamiento antiagregante dual es limitado y controversial. Un meta-análisis de Burger y cols, estudió el impacto de aspirina en el sangrado quirúrgico, mostrando un incremento en $50 \%$ sin aumento de morbi-mortalidad, con excepción de neurocirugía y resección transuretral de próstata ${ }^{20}$. En cirugía ortopédica los resultados son contradictorios entre mayor sangrado en artroplastía de cadera pero no en fractura de cuello de fémur ${ }^{21}$.

Chassot y cols, en una revisión reciente estiman que las pérdidas sanguíneas aumentan entre 2,5$20 \%$ por aspirina y $30-50 \%$ por clopidogrel. Ellos encontraron que la necesidad de transfusiones aumentó $30 \%$, pero la mortalidad no aumentó, excepto nuevamente en cirugía intracraneana y resección transuretral de próstata ${ }^{22}$.

Datos aportados por Jolly y $\mathrm{cols}^{23}$ sugieren que la combinación de clopidogrel $300 \mathrm{mg}$ y aspirina en bajas dosis $(<100 \mathrm{mg})$ es tan efectiva en la prevención de eventos isquémicos comparada con dosis $>200 \mathrm{mg}$, acompañándose de menor incidencia de sangrado.

Múltiples reportes de casos describen hemorragias quirúrgicas significativas e inclusos fatales con el uso de terapia dual en cirugía vascular, ortopédica e incluso procedimientos endoscópicos ${ }^{43}$. Sin embargo, a pesar de que muchos estudios han demostrado mayor sangrado, no han sido consistentes en mostrar mayor mortalidad ni cambios en los resultados quirúrgicos ${ }^{21,22,40,43}$.

De la literatura se desprende que existen distintos grupos de cirugías según riesgo de hemorragia $\left(\right.$ Tabla 2) ${ }^{32}$. Así es como se recomienda mantener tratamiento dual, salvo en cirugía con alto riesgo de hemorragia, como en cavidades cerradas (intracraneana, retinal, espinal), manteniendo el uso de aspirina y suspendiendo clopidogrel 7 días antes de la cirugía.

La evidencia sugiere que en pacientes con SC puestos recientemente, el riesgo de sangrado es despreciable en relación al beneficio de mantener el tratamiento antiplaquetario. 
Tabla 2. Riesgo de hemorragia en cirugía no cardiaca

\begin{tabular}{|ll|}
\hline Riesgo & Tipo de cirugías \\
\hline Alto & Neurocirugía intracraneal \\
& $\begin{array}{l}\text { Cirugía canal espinal } \\
\text { Cirugía ocular cámara posterior }\end{array}$ \\
& Cirugía abdominal \\
& Vascular \\
& Ortopédica mayor \\
& Otorrinolaringología \\
& Urología \\
Bajo & Plástica periférica \\
& Ortopédica menor \\
& Dental \\
& Endoscópica \\
& Biopsias \\
\hline
\end{tabular}

\section{Recomendaciones para el manejo perioperatorio}

La mayor duda hasta ahora es establecer la duración óptima del tratamiento antiplaquetario dual. No existen estudios controlados aleatorios en relación al manejo perioperatorio de pacientes con SC, todos son reportes de casos clínicos.

Las estrategias de manejo deben basarse en los riesgos individuales de trombosis o de hemorragia, influidos por la urgencia, tipo y localización de la cirugía y decidido de acuerdo con todo el equipo médico y el paciente $e^{17,25,32}$. Las decisiones más importantes deben incluir: momento para realizar la cirugía, mantener o suspender los antiagregantes o iniciar terapia "puente".

\section{Terapia "puente" en cirugía no cardiaca}

Debe considerarse terapia "puente" en casos de alto riesgo de trombosis del SC así como en cirugías con alto riesgo de hemorragia ${ }^{24}$.

El uso de heparinas de cualquier tipo ha sido descrita, sin embargo, no hay evidencia claras de que sean siempre efectivas, fundamentalmente porque no tienen actividad antiplaquetaria ${ }^{27}$. Webster y cols mostraron en cirugía vascular que la heparina incluso es capaz de aumentar la agregación plaquetaria en pacientes que usan aspirina ${ }^{2}$.

Las recomendaciones francesas sugieren que en pacientes en los que se hubiere utilizado terapia dual, la suspensión del clopidogrel se debe acompañar de terapia puente con AINEs y heparina de bajo peso molecular. Este esquema, sin embargo, no ha probado efectividad ${ }^{26}$.

En 2007 Broad y cols publicaron una pequeña serie con SM en pacientes de alto riesgo de trombosis, a los cuales se les mantuvo aspirina y se suspendió clopidogrel 5 días previos a la cirugía, usándose heparina y tirofiban (inhibidor de GP IIb/IIIa) en infusión hasta 8 h antes de la cirugía. No se registraron casos de hemorragia ni de trombosis de $\mathrm{SC}^{27}$.

Recientemente, Savanitto y cols publicaron el uso de tirofiban como "puente" en cirugía de urgencia en pacientes con menos de un año instalación de SM y alto riesgo de trombosis, concluyendo que su uso era seguro en relación al riesgo de hemorragia ${ }^{28}$.

Actualmente no hay datos suficientes para recomendar una terapia "puente" que mejore los resultados especialmente en pacientes de alto riesgo de trombosis de SC en el post operatorio inmediato, sin embargo parece atractiva la alternativa de inhibidores de GP IIb/IIIa por su corta duración (50\% actividad plaquetaria a 4 horas de suspensión $)^{31}$.

\section{Manejo perioperatorio recomendado}

La AHA concluye que la suspensión prematura del tratamiento antiplaquetario dual aumenta marcadamente el riesgo de trombosis de SC. Recomiendan posponer toda cirugía electiva que tenga riesgo de hemorragia hasta que el período de tratamiento antiagregante recomendado se complete. Si es posible, debe mantenerse aspirina y suspenderse clopidogrel, para reiniciarse lo antes posible en el postoperatorio. En caso de cirugía electiva después de la inserción de un SNM debe realizarse entre la $6^{a}$ y $12^{a}$ semana y después de un SM debe esperarse un año ${ }^{17-25}$.

Para cirugías de urgencia no es posible esperar, la sugerencia es mantener el tratamiento antiplaquetario dual, pero si el riesgo de sangrado es muy alto debe suspenderse y reiniciarse prontamente en el postoperatorio ${ }^{29}$. Esto es aplicable a todas las 


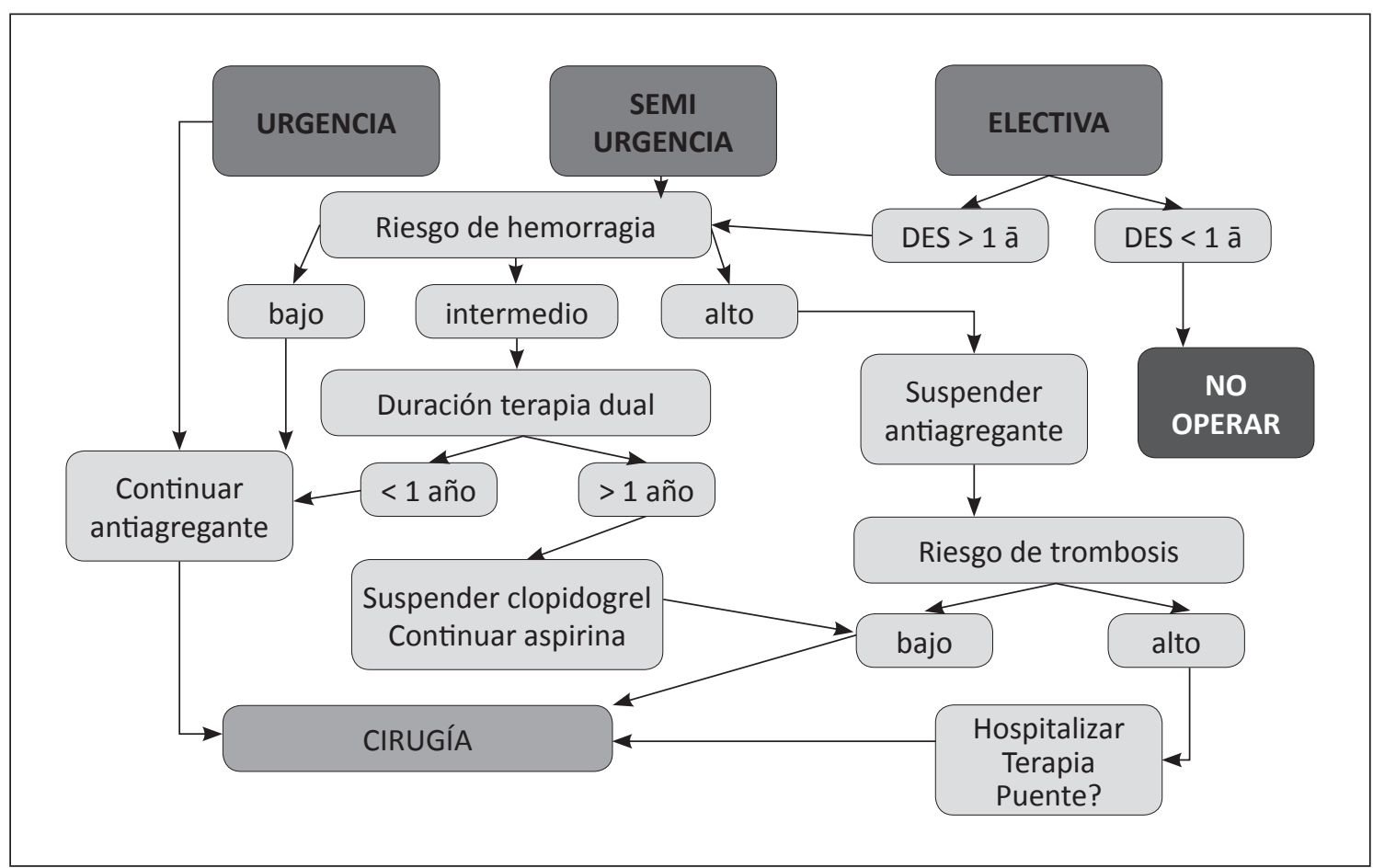

Figura 1. Algoritmo del manejo de pacientes con "Drug Eluting Stents (DES)" y cirugía no cardiaca. Adaptado de Abualsaud A., Eisenberg M. Perioperative Management of Patients With Drug Eluting Stents, JACC Intv. 2010; 3: 135.

cirugías, excepto en cirugías de espacios cerrados o donde podría ser catastrófico, como intracraneal, intraespinal o cámara ocular posterior ${ }^{32,40}$, en estos casos es admisible el uso de alguna terapia puente $^{27,28,32}$ (Figura 1).

Dado el riesgo de trombosis los SC, estos pacientes deben manejarse en una unidad de alto cuidado, con monitoreo de ECG continuo y medición diaria de enzimas cardiacas.

\section{Manejo de la trombosis del stents coronario y de hemorragia quirúrgica}

La trombosis de un SC se manifiesta con angor, supradesnivel del segmento ST, hipotensión refractaria, arritmias ventriculares y muerte súbita. $\mathrm{Si}$ se sospecha, debe actuarse inmediatamente. El tratamiento trombolítico es poco efectivo y está contraindicado en el postoperatorio; siendo la ICP la terapia de elección ${ }^{30}$.

Debe observarse cuidadosamente la posibilidad de hemorragia mayor en estos pacientes y hay que estar preparado para la eventual terapia de reemplazo con plaquetas ${ }^{17}$, no existiendo un rol de trasfusiones de plaquetas profilácticas.

\section{Conclusiones}

El manejo de los pacientes con SC presenta riesgos importantes. Algunas preguntas siguen sin respuesta por la inexistencia de estudios controlados prospectivos randomizados que definan el manejo de estos pacientes. Las decisiones necesitan de una adecuada discusión caso a caso entre el cardiólogo, el anestesiólogo y el cirujano, instancia en la que cual se debe evaluar el riesgo individual de trombosis del SC versus el riesgo de sangramiento quirúrgico. Para cirugía electiva la suspensión del tratamiento antiagregante debe retrasarse hasta que haya pasado el riesgo de trombosis establecido en las recomendaciones, siendo de 4 a 6 semanas posterior a un SNM y a un año como mínimo post $\mathrm{SM}$, considerando los factores de riesgo que pueden hacer recomendable prolon- 
gar la terapia. En caso de cirugía de urgencia antes del período mínimo del tratamiento recomendado, idealmente la terapia dual debe mantenerse en el perioperatorio, pero debe balancearse con el riesgo de hemorragia.

Si la terapia antiagregante plaquetaria dual debe suspenderse, esta debería reiniciarse a la brevedad posible después de la cirugía. Deben esperarse los resultados de nuevos estudios para ver el rol de las terapias "puente". Los pacientes deben tener monitorización completa para sangramiento quirúrgico y para trombosis del SC, idealmente en una unidad de paciente crítico.

Hay estudios con nuevas generaciones de SC, los que sugieren por ahora endotelización retardada. En la actualidad los pacientes con SC de primera generación deben seguir siendo manejados cuidadosamente y caso a caso.

\section{Referencias}

1. Lloyd-Jones, Adame R, Carnethon M. Heart diseases and stroke stadistics 2009 up date. Circulation 2009; 119: e21-e181.

2. Newsome LT, Weller RS, Gerancher JC. Coronary stents: perioperative considerations and Management. Anesth Analg 2008; 107: 570-90.

3. Serruys PW, Kutryk MJ, Ong AT, Metzler H., James SK. Coronary-artery stents. NEngl JMed 2006; 354: 483-95.

4. Cuttip DF, Balm DS, Ho K, Popma J, Lnasky A, Cohen $\mathrm{D}$, et al. Stent trombosis in the modern era: analisis of multicenter coronary stent clinical trials. Circulation 2001; 103: 1967-71.

5. Hoffman R, Mintz GS. Coronary in-stent restenosispredictors, treatment and prevention. Eur Heart J 2000; 21: 1739-49.

6. Stone GW, Moses JW, Ellis S, Schofler J, Dawkins K, Morice M, et al. Safety and efficacy of sirolimus and paclitaxel eluting coronary stents. N Engl J Med 2007; 356: 998-1008.

7. Newsome L, Kutcher M, Royster R. Coronary artery stents: part 1. Evolution of percutaneous coronary intervention. Anesth Analg 2008; 107: 552-69.

8. Iakovou I, Schmidt T, Bonizzoni E, Ge L, Snagiorgo G, Stankovic G, et al. Incidence, predictors, and outcome of thrombosis after successful implantation of drug-eluting stents. JAMA 2005; 293: 2126-30.

9. Rodríguez AE, Mieres J, Fernández -Pereira C, Jiménez A. Coronary stent thrombosis in the current drug-eluting stent era: insights from the ERACI III trial. JAmColl
Cardiol 2006; 47: 205-7.

10. Win HK, Caldera A, Maresh K, López J, Rihal C, Parikh $\mathrm{M}$, et al. Clinical outcomes and stent thrombosis following off- label use of drug-eluting stents. JAMA 2007; 297: 2001-9.

11. Jiminez AH, Stubbs ME,Tofler G, Winther K, Williams G, Muller J, et al. Rapidity and duration of platelet suppression by enteric-coated aspirin in healthy young men. Am J Cardiol 1992; 69: 258-62.

12. Ho PM, Peterson ED, Wang L. Incidence of death and acute myocardial infarction associated with stopping clopidogrel after acute coronary syndrome. JAMA 2008; 299: 532-9.

13. Mahla E, Lang T, Vicenzi M, Werkgartner G, Chia SH. Thromboelastography for monitoring prolonged hypercoaguability after major abdominal surgery. Anesth Analg 2001; 92: 572.

14. Sharma AK, Ajani AE, Hamwi S, Maniar P, Lakhani S, Walsman R, et al. Major non-cardiac surgery following coronary stenting. When is it safe to operate? Catheter Cardiovasc Interv 2004; 63: 141-5.

15. Wilson SH, Fasseas P, Orford J, Lennon R, Horlocker T, Charnoff $\mathrm{N}$, et al. Clinical outcome of patients undergoing non-cardiac surgery in the two months following coronary stenting. J Am Coll Cardiol 2003; 42: 234-40.

16. Banerjee S, Varghese C, Samuel J, Eikelboom JW. Comparison of the impact of short and long-term Clopidogrel use following percutaneous coronary intervention on mortality. AmJ Cardiol 2008; 102: 1159-62.

17. Fleisher LA, BeckmanJA, BrownKA, Calkins H, Chaikof E, Fleishermann K, et al. ACC/AHA2007guidelines on perioperative cardiovascular evaluation and care for noncardiac surgery: a report of the American College of Cardiology/American Heart Association. J Am Coll Cardiol 2007; 50: el 59-241.

18. Nuttall GA, Brown MJ, Stornbaugh J, Michon P, Hathaway $\mathrm{M}$, Lindeen $\mathrm{K}$, et al. Time and cardiac risk of surgery after bare-metal stent percutaneous coronary intervention. Anesthesiology 2008; 109: 588-95.

19. Rabbitts JA, Nuttall GA, Brown MJ, Schouten O, Moen MD. Cardiac risk of noncardiac surgery after percutaneous coronary intervention with drug-eluting stents. Anesthesiology 2008; 109: 596-604.

20. Burger W, Chemnitius JM, Kneissi G, Rucker G. Lowdose aspirin for cardiovascular prevention, cardiovascular risks after its preoperative withdrawal versus bleeding risks with its continuation. J Int Med 2005; 257: 399-414.

21. Nuttal GA, Santrach PJ, Oliver Jr WC, Horlocker T, Shaughnessy W, Cabanela M, et al. The predictors of blood transfusions in total hip arthroplasties. Transfusion 1996; 36: 144-9. 
22. Chassot PG, Delabays A, Spahn DR. Perioperative antiplatelet therapy: the case for continuing therapy in patients at risk of myocardial infarction. Br J Anaesth 2007; 99: 316-28.

23. Jolly SS, Pogue J, Haladyn K, Hirsh J. Effects of aspirin dose on ischaemic events and bleeding after percutaneous coronary intervention: insights from PCICUREstudy. Eur Heart J2009; 30: 900-7.

24. Dalal AR, D'Souza S, Shulman MS. Brief review: coronary drug-eluting stents and anesthesia. Can J Anaesth 2006; 53: 1230-43.

25. Practice alert for the perioperative management of patients with coronary artery stents: a report by the American Society of Anesthesiologists Committee on Standards and Practice Parameters. Anesthesiology 2009; 110: 22-41.

26. Albaladejo P, Marret E, Piriou V, Samana C. French Society of Anaesthesiology and Intensive Care. Perioperative management of antiplatelet agents in patients with coronary stents: recommendations of a French task force. Br J Anaesth 2006; 97: 580-2.

27. Broad L, Lee T, Conroy M, Bolsin S, Orford N, Black A, et al. Successful management of patients with a drugeluting coronary stent presenting for elective noncardiac surgery. Br J Anaesth 2007; 98: 19-22.

28. Savanitto S, D’Urbano M, Caracciolo M, Barlocco F, Mariani G, Nichelatti M, et al. Urgent surgery in recently implanted coronary stent: study of bridging antiplaquelet therapy with Tirofiban. Br J Anesth 2010; 104: 285-91.

29. Augoustides JG. Perioperative thrombotic risk of coronary artery stents: possible role for intravenous platelet blockade. Anesthesiology 2007; 07: 516.

30. Brilakis ES, Banerjee S, Berger PB. Perioperative management of patients with coronary stents. J Am Coll Cardiol 2007; 49: 2145-50.

31. Moen MD, Keating GM, Wellington K. Bivalirudin: are view of its use in patients undergoing percutaneous coronary intervention. Drugs 2005; 65: 1869-91.

32. Abaualsaud A, Eisenberg M. Perioperative management of patients with drug-eluting stents. J Am Coll Cardiol Intv 2010; 3: 131-42.

33. Hodgson J, Stone G, Lincoff A. Late stent thrombosis: considerations and practical advice for the use of drugeluting stents: a report from the Society for Cardiovascular Angiography and Interventions Drug-eluting Stent Task Force. Catheter Cardiovasc Interv 2007; 69: 327-33.

34. Eisentein E, Anstron K, Kong D. Clopidogrel use and long term clinical outcome after DES implementation. JAMA 2007; 297: 159-68.

35. De la Torre P, Hernández J, Alfonso F, San Martín M, Pinar M, Elizaga F. Drug eluting stents trombosis, results from multicenter Spanish registry. Am Coll Cardiol 2008; 51: 986-96.

36. Eisenberg M, Richard P, Liberman D, Filisen K. Safety of short term discontinuation of antiplatelet therapy in patients with DES. Circulation 2009; 119: 1634-42.

37. Kuchulakach P, Chu W, Tsuchida R. Correlates and long term outcome of angiographically proven stent trombosis. Circulation 2006; 113: 1108-13.

38. Wildecker S, Meirer B. Late coronary stent trombosis. Circulation 2007; 116: 1952-65.

39. Park D, Park S, Colombo T. Frecuency of and risk for trombosis after DES implantation during long follow up. Am J Cardiolo 2006; 98: 352-66.

40. Burger W, Chemnitius J, Kneissl G, Rucker G. Low-dose aspirin for secondary cardiovascular prevention-cardiovascular risks after its perioperative withdrawal versus bleeding risks with its continuation. J Intern Med 2005; 257: 399-414.

41. Srinivasan AK, Grayson AD, Pullan DM, Fabri BM, Dihmis WC. Effect of preoperative aspirin use in off-pump coronary artery bypass operations. Ann Thorac Surgery 2003; 76: 41-5.

42. Leong JY, Baker RA, Shah PJ, Cherian VK, Knight JL. Clopidogrel and bleeding after coronary artery bypass graft surgery. Ann Thorac Surg 2005; 80: 928-33.

43. Moore M, Power M. Perioperative hemorrhage and combined clopidogrel and aspirin therapy. Anesthesiology 2004; 101: 792-4. 